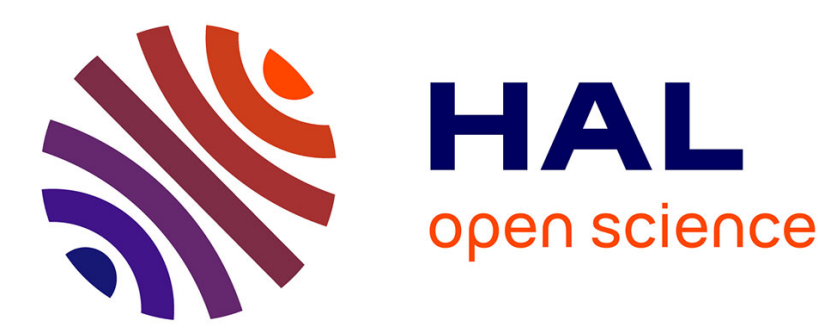

\title{
Kinematic Modeling of an EAP Actuated Continuum Robot for Active Micro-endoscopy.
}

Mohamed Taha Chikhaoui, Kanty Rabenorosoa, Nicolas Andreff

\section{To cite this version:}

Mohamed Taha Chikhaoui, Kanty Rabenorosoa, Nicolas Andreff. Kinematic Modeling of an EAP Actuated Continuum Robot for Active Micro-endoscopy.. Advances in Robot Kinematics., 2014, pp.457-465. 10.1007/978-3-319-06698-1_47. hal-01051375

\section{HAL Id: hal-01051375 \\ https://hal.science/hal-01051375}

Submitted on 25 Jul 2014

HAL is a multi-disciplinary open access archive for the deposit and dissemination of scientific research documents, whether they are published or not. The documents may come from teaching and research institutions in France or abroad, or from public or private research centers.
L'archive ouverte pluridisciplinaire $\mathbf{H A L}$, est destinée au dépôt et à la diffusion de documents scientifiques de niveau recherche, publiés ou non, émanant des établissements d'enseignement et de recherche français ou étrangers, des laboratoires publics ou privés. 


\title{
Kinematic modeling of an EAP actuated continuum robot for active micro-endoscopy
}

\author{
Mohamed Taha Chikhaoui, Kanty Rabenorosoa, and Nicolas Andreff
}

\begin{abstract}
An active micro-endoscope based on concentric tubes, an emerging class of continuum robots, is presented hereby. It is designed to reach the digestive tube and the stomach for early cancer detection and intervention. The manipulator is constructed from three flexible, telescopic, and actuated tubes. The actuators are based on Electro-Active Polymer electrodes coated and patterned around the tube. A full multi-section kinematic model is developed; it is used to compare the existing constant curvature configuration to the proposed micro-endoscope. That comparison is established according to the reachable workspace and the performance indices. The results are used to prove the effectiveness of the embedded actuation method to reach the workspace more dexterously, which is very useful in medical systems, especially in surgical applications.
\end{abstract}

Key words: Continuum robot, Active cannula, Electro-Active Polymers (EAP), Kinematic modeling

\section{Introduction}

Continuum robots are still enthralling researchers' interests, almost half a century after the first early prototype: the "Tensor Arm" of [1]. Exceptional usefulness of continuum robots appears in applications where it is restrictive to have joints and stiff links. They have the potential to suffer localized damage while still maintaining a healthy degree of functionality [4]. Moreover, continuum robots are able to navigate through complex anatomy. They may be considered as part of robots for MIS (Minimally Invasive Surgery) and NOTES (Natural Orifice Transluminal Endoscopic Surgery). Recent medical continuum robots include laparoscopic application,

Mohamed T. Chikhaoui · Kanty Rabenorosoa $\cdot$ Nicolas Andreff

Automatic Control and Micro-Mechatronics Department, FEMTO-ST Institute, Besançon, France e-mail: firstname.lastname@ femto-st.fr 
laser manipulators, catheters and micro-endoscopes, summarized in [9]. Concentric tubes developed by $[7,10]$ for endonasal skull base surgery are a major contribution to continuum robots category. Such manipulators are constructed from three precurved telescopic concentric tubes that have small diameters (less than $3 \mathrm{~mm}$ ). They are actuated at their base by translation and axial rotation of each tube, and the overlapping builds the final shape.

Concentric tubes are the starting point of our study. We aim to change tubes curvatures by the means of embedded actuators, and thereby, provide additional degrees of freedom to the system. Monitoring the curvature and bending each tube in different directions are indeed expected to enhance the manipulator performances. Our considered manipulator is presented in Figure 1a, showing a CAD design of a curved tube that holds a laser tool at its end-effector. The bending is performed with Electro-Active Polymer (EAP) electrodes coated around the tube based on [8] work and sketched in Figure 1b.

a)

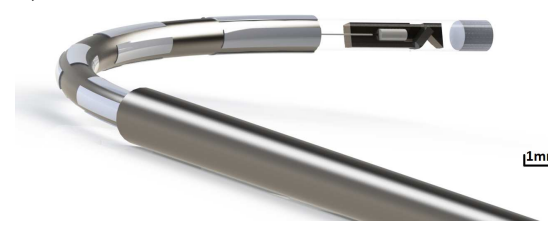

b)

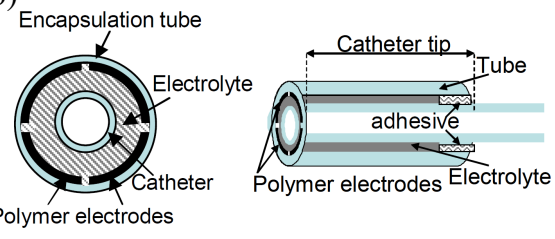

Fig. 1: Design of the considered continuum robot. a) CAD design of the tubes, b) Illustration of an EAP-based embedded actuation [8].

We present in the next section the kinematic modeling of an active cannula robot, starting from a standard approach to achieve modeling of an EAP actuated continuum manipulator. In section 3, both models will be used to analyze a part of the reachable workspace. Then we can establish a theoretical comparison between the existing configurations and our design, using also performance indices to confirm the manipulability improvement. Finally, in section 4, we will conclude and present several future challenges that still need to be achieved in this field.

\section{Kinematic modeling}

Before we can start modeling differential kinematics, a few assumptions need to be set up in the standard approach. This concerns the description used in previous work [10]. Furthermore, the modeling of a continuum robot with EAP-based embedded actuators will be described. 


\subsection{Standard approach}

\subsubsection{Arc parameters}

The standard approach [10] is based on a configuration of $n$ concentric tubes presented in Figure 2a. Each tube $i \in\{1 . . n\}$ is made of a straight part $\left(S_{i}\right)$ and a precurved distal one $\left(C_{i}\right)$ and can translate (by $\rho_{i}$ ) and rotate (by $\theta_{i}$ ) with respect to the $z$-axis of the robot base. Thus, depending on the translation of each tube, the concentric assembly can be decomposed into $m$ successive links, defined by the concentric overlaps of straight parts, precurved parts or nothing (e.g. for three tubes, $C_{3} / S_{2} / S_{1}$ or $C_{3} / C_{2} / \emptyset$ ). Each link is modeled by an arc of a circle (constant curvature assumption), described by three parameters: its length $\ell_{j}$, its curvature $\kappa_{j}$ which is the inverse of the radius of curvature $r_{j}$, and the angle $\phi_{j}$ of the so-called equilibrium plane containing the arc (Figure 2b).

Depending on the overlapping of the $n$ tubes, the curvature of link $j \in\{1, \ldots, m\}$ is given by:

$$
\kappa_{j}=\sqrt{\kappa_{x_{j}}^{2}+\kappa_{y_{j}}^{2}} \quad \text { with } \quad \kappa_{x_{j}}=\frac{\sum_{i=1}^{n} E_{i} I_{i} \kappa_{i, j} \cos \theta_{i, j}}{\sum_{i=1}^{n} E_{i} I_{i}}, \kappa_{y_{j}}=\frac{\sum_{i=1}^{n} E_{i} I_{i} \kappa_{i, j} \sin \theta_{i, j}}{\sum_{i=1}^{n} E_{i} I_{i}}
$$

where $E_{i}$ is the elastic modulus, $I_{i}$ is the cross sectional moment of inertia, $\kappa_{i, j}$ is the intrinsic curvature of the $i^{\text {th }}$ tube in the $j^{\text {th }}$ link and $\theta_{i, j}$ denotes the $i^{\text {th }}$ tube angle about the $j^{\text {th }}$ link frame $z$-axis. Finally, the equilibrium plane angle is given by:

$$
\phi_{j}=\arctan \left(\kappa_{y_{j}} / \kappa_{x_{j}}\right)
$$

a)

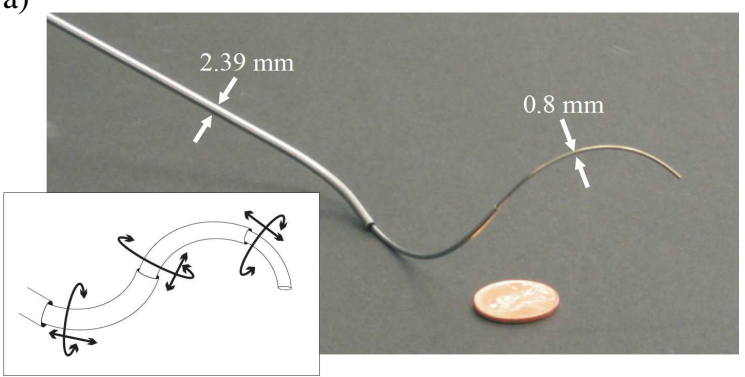

b)

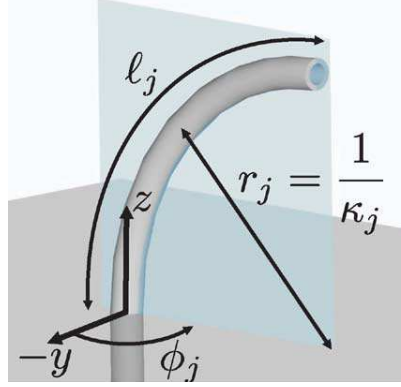

Fig. 2: Active cannula description: a) three concentric tube configuration [9], and b) arc parameter description [11].

\subsubsection{Specific mapping and independent mapping}

In [11], three spaces are defined: 
- the actuator space: $\left\{\rho_{i}, \theta_{i} \mid i \in\{1 . . n\}\right\}$

- the configuration space: $\left\{\kappa_{j}, \phi_{j}, \ell_{j} \mid j \in\{1 . . m\}\right\}$

- the task space: $S E(3)$

Two space transformations are thus defined:

1. The specific mapping from the actuator space to the configuration space (actuator dynamics). This mapping totally depends on the actuation of the tubes.

2. The independent mapping from the configuration space to the task space (forward kinematics). This mapping is the same for all concentric tube architectures, satisfying the assumption of constant curvature links and can be generically modeled.

Forward kinematics can be accomplished in a variety of ways: through DenavitHartenberg parameters [5], Frenet-Serret frames [5], integral formulation [3], and exponential coordinates [7] and [11]. Using the latter convention, the transformation $T_{j}$ from link $j-1$ to link $j$ decomposes into a rotation of center $\mathbf{r}_{\mathbf{j}}=\left[1 / \kappa_{j}, 0,0\right]^{T}$ about the $y$ axis by $\alpha_{j}$ and a rotation about the $z$ axis by $\phi_{j}$ :

$$
T_{j}=\left[\begin{array}{cc}
R_{z}\left(\phi_{j}\right) & 0 \\
0 & 1
\end{array}\right]\left[\begin{array}{cc}
R_{y}\left(\alpha_{j}\right) \mathbf{p}_{\mathbf{j}} \\
0 & 1
\end{array}\right]
$$

where $\alpha_{j}=\kappa_{j} \ell_{j}$ and $\mathbf{p}_{\mathbf{j}}=\left[r_{j}\left(1-\cos \alpha_{j}\right), 0, r_{j} \sin \alpha_{j}\right]^{T}$.

\subsubsection{Differential kinematics}

To compute constant curvature kinematics of a multi-section tube, one must compute single section tube kinematics. For the brevity of this paper, the computation is omitted but details can be found in [9]. The velocity of link $j$ with respect to link $j-1$ is given by:

$$
\mathbf{V}_{\mathbf{j}}=\underbrace{\left[\begin{array}{cccc}
\cos \Delta \phi_{j}\left(\cos \left(\kappa_{j} \ell_{j}\right)-1\right) / \kappa_{j}^{2} & 0 & 0 \\
\sin \Delta \phi_{j}\left(\cos \left(\kappa_{j} \ell_{j}\right)-1\right) / \kappa_{j}^{2} & 0 & 0 \\
-\left(\sin \left(\kappa_{j} \ell_{j}\right)-\kappa_{j} \ell_{j}\right) / \kappa_{j}^{2} & 0 & 1 \\
-\ell_{j} \sin \Delta \phi_{j} & 0-\kappa_{j} \sin \Delta \phi_{j} \\
\ell_{j} \cos \Delta \phi_{j} & 0 & \kappa_{j} \cos \Delta \phi_{j} \\
0 & 1 & 0
\end{array}\right]}_{J_{j}}\left[\begin{array}{c}
\dot{\kappa}_{j} \\
\dot{\phi}_{j} \\
\dot{\ell}_{j}
\end{array}\right] \quad \text { where } \Delta \phi_{j}=\phi_{j}-\phi_{j-1}
$$

Using the adjoint transformation introduced by [6], the full independent kinematic Jacobian can be deduced from the individual ones:

$$
J_{\text {indep }}=\left[\begin{array}{llll}
J_{0} & A d_{T_{0}} J_{1} A d_{T_{01}} J_{2} \ldots A d_{T_{0(m-1)}} J_{m}
\end{array}\right]
$$

where $T_{0 j}=T_{0} T_{1} \ldots T_{j}$ is the $j^{\text {th }}$ transformation matrix at the $j^{\text {th }}$ link and

$$
A d_{T}=\left[\begin{array}{cc}
R & R[t]_{\times} \\
0 & R
\end{array}\right]
$$


with $R$ and $\mathbf{t}$ the rotation and translation component of $T$ and $[t]_{\times}$the skewsymmetric matrix associated to the vector cross-product by $\mathbf{t}$. Thus, determining the number of links in a configuration is a preliminary task to understand full Jacobian matrix dimension. For a configuration with three totally curved concentric tubes, we obtain a 6 by 9 matrix, as there are 3 links.

The specific kinematic Jacobian maps actuator derivatives $\left[\begin{array}{ll}\dot{\rho}_{i} & \dot{\theta}_{i}\end{array}\right]^{T}$ into arc parameters derivatives $\left[\begin{array}{lll}\dot{\kappa}_{j} & \Delta \dot{\phi}_{j} & \dot{\ell}_{j}\end{array}\right]^{T}$. In the three totally curved concentric tube configuration, the full specific kinematic Jacobian $J_{\text {spec }}$ is a 3 by 2 matrix. Consequently, this configuration is a non-holonomic robot: the whole space can be reached but, in a given state, only a subset of velocity directions is achievable.

\subsection{Modeling of EAP actuated concentric tubes}

Adding an embedded actuation to the previous configuration is beneficial as it provides a direct control of the intrinsic curvatures $\kappa_{i, j}$ of each tube, whereas the standard approach only takes into account constant intrinsic precurvatures. This adds one component per tube in the actuator space $\left(\rho_{i}, \theta_{i}, \kappa_{i}\right)$ but does not change the two other spaces. Therefore, the independent mapping and the independent kinematic Jacobian are the same as those described above, and only the specific mapping and specific kinematic Jacobian need to be derived.

The curvature of a tube made of Electro-Active Polymer (Figure 1) follows a linear law in terms of voltage according to the relation explained in [8]:

$$
\kappa_{i, j}=1 / r_{i, j}=C_{P P Y} V_{i},
$$

where $C_{P P Y}$ is considered as the Polypyrrole actuation constant and $V_{i}$ is the applied voltage.

By appropriate low-level control, we foresee to be able to servo $\kappa_{i, j}$, the intrinsic precurvature of tube $i$ in link $j$, to a desired value. Thereby, the specific mapping will have the same expression as in the standard approach, with only a change in the inputs (precurvatures). To compute the dependent Jacobian, for three totally curved concentric tubes, we first need to determine the number of links. With such configuration, there are three links: three tubes for the first link, two tubes for the second link, and one tube for the third link as shown in Figure 3a.

Differentiating (1) with respect to $\dot{\kappa}_{i, j}$ and $\dot{\theta}_{i, j}$ yields:

$$
\dot{\kappa}_{j}=\frac{1}{\sqrt{\kappa_{x_{j}}^{2}+\kappa_{y_{j}}^{2}}}\left[\begin{array}{ll}
\kappa_{x_{j}} & \kappa_{y_{j}}
\end{array}\right]\left[\begin{array}{ll}
\mathbf{A}_{\mathbf{j}} & \mathbf{B}_{\mathbf{j}} \\
\mathbf{C}_{\mathbf{j}} & \mathbf{D}_{\mathbf{j}}
\end{array}\right]\left[\begin{array}{l}
\dot{\kappa}_{i n, j} \\
\dot{\theta}_{i n, j}
\end{array}\right]
$$

where $\dot{\kappa}_{\mathbf{i n}, \mathbf{j}}=\left[\dot{\kappa}_{1, j} \dot{\kappa}_{2, j} \dot{\kappa}_{3, j}\right]^{T}, \dot{\theta}_{\mathbf{i n}, \mathbf{j}}=\left[\dot{\theta}_{1, j} \dot{\theta}_{2, j} \dot{\theta}_{3, j}\right]^{T}, \dot{\kappa}_{i}=C_{P P Y} . \dot{V}_{i}$ and 


$$
\begin{aligned}
\mathbf{A}_{\mathbf{j}} & =\left[\begin{array}{lll}
\frac{E_{1} I_{1} \cos \theta_{1, j}}{\Sigma_{i} E_{1} I_{1}} & \frac{E_{2} I_{2} \cos \theta_{2, j}}{\Sigma_{i} E_{2} I_{2}} & \frac{E_{3} I_{3} \cos \theta_{3, j}}{\Sigma_{i} E_{3} I_{3}}
\end{array}\right] \\
\mathbf{B}_{\mathbf{j}} & =\left[\begin{array}{lll}
-\frac{E_{1} I_{1} \kappa_{1, j} \sin \theta_{1, j}}{\Sigma_{i} E_{1} I_{1}}-\frac{E_{2} I_{2} \kappa_{2, j} \sin \theta_{2, j}}{\Sigma_{i} E_{2} I_{2}}-\frac{E_{3} I_{3} \kappa_{3, j} \sin \theta_{3, j}}{\Sigma_{i} E_{2} I_{2}} &
\end{array}\right] \\
\mathbf{C}_{\mathbf{j}} & =\left[\begin{array}{lll}
\frac{E_{1} I_{1} \sin \theta_{1, j}}{\Sigma_{i} E_{1} I_{1}} & \frac{E_{2} I_{2} \sin \theta_{2, j}}{\Sigma_{i} E_{2} I_{2}} & \frac{E_{3} I_{3} \sin \theta_{3, j}}{\Sigma_{i} E_{3} I_{3}}
\end{array}\right] \\
\mathbf{D}_{\mathbf{j}} & =\left[\begin{array}{lll}
\frac{E_{1} I_{1} \kappa_{1, j} \cos \theta_{1, j}}{\Sigma_{i} E_{1} I_{1}} & \frac{E_{2} I_{2} \kappa_{2, j} \cos \theta_{2, j}}{\Sigma_{i} E_{2} I_{2}} & \frac{E_{3} I_{3} \kappa_{3, j} \cos \theta_{2, j}}{\Sigma_{i} E_{2} I_{2}}
\end{array}\right]
\end{aligned}
$$

Similarly, differentiating (2) yields:

$$
\dot{\phi}_{j}=\frac{1}{\sqrt{\kappa_{x_{j}}^{2}+\kappa_{y_{j}}^{2}}}\left[-\kappa_{y_{j}} \kappa_{x_{j}}\right]\left[\begin{array}{ll}
\mathbf{A}_{\mathbf{j}} & \mathbf{B}_{\mathbf{j}} \\
\mathbf{C}_{\mathbf{j}} & \mathbf{D}_{\mathbf{j}}
\end{array}\right]\left[\begin{array}{c}
\dot{\kappa}_{i n, j} \\
\dot{\theta}_{i n, j}
\end{array}\right]
$$

For the $j^{\text {th }}$ link, the corresponding arc length derivative corresponds to $\dot{\ell}_{j}=\dot{\rho}_{j}$. This leads to the specific kinematic Jacobian at the $j^{\text {th }}$ link:

$$
\left[\begin{array}{c}
\dot{\kappa}_{j} \\
\dot{\phi}_{j} \\
\dot{\ell}_{j}
\end{array}\right]=\frac{1}{\sqrt{\kappa_{x_{j}}^{2}+\kappa_{y_{j}}^{2}}}\left[\begin{array}{ccc}
\kappa_{x_{j}} \mathbf{A}_{\mathbf{j}}+\kappa_{y_{j}} \mathbf{C}_{\mathbf{j}} & \kappa_{x_{j}} \mathbf{B}_{\mathbf{j}}+\kappa_{y_{j}} \mathbf{D}_{\mathbf{j}} & 0 \\
-\kappa_{y_{j}} \mathbf{A}_{\mathbf{j}}+\kappa_{x_{j}} \mathbf{C}_{\mathbf{j}}-\kappa_{y_{j}} \mathbf{B}_{\mathbf{j}}+\kappa_{x_{j}} \mathbf{D}_{\mathbf{j}} & 0 \\
0 & 0 & \sqrt{\kappa_{x_{j}}^{2}+\kappa_{y_{j}}^{2}}
\end{array}\right]\left[\begin{array}{c}
\dot{\kappa}_{i n, j} \\
\dot{\theta}_{i n, j} \\
\dot{\rho}_{j}
\end{array}\right]
$$

The above specific kinematic Jacobian is a square matrix and does not contain any structural non-holonomic constraint. Moreover, it is a 3 by 3 matrix. Consequently, only two tubes should be enough to reach any pose in the workspace. However, keeping three tubes provides us with a redundancy which is highly recommended, especially in medical and surgical manipulators.

\section{Performance analysis}

\subsection{Workspace analysis}

Workspace means the reachable zones of a manipulator end-effector. In traditional robotics, one can obtain the workspace by inverting the direct geometrical model. However, in continuum robotics, converting the effect of curvature change or bending angle into movements is significantly more complex. We restrict our analysis to planar movements for an intermediate step. This is sufficient to illustrate the key advantages of changing an additional arc parameter: the curvature.

We take into account a simple configuration case: three tubes with the same insertion angle $\alpha_{i}$ that are not rotated about their $z$ axis. The total robot length is $45 \mathrm{~mm}$ and the tube outer diameters are respectively $3.05 \mathrm{~mm}, 1.45 \mathrm{~mm}$ and $0.72 \mathrm{~mm}$. The only possibility for a precurved concentric tube configuration to reach additional zones in the $x-z$ plan is to combine tube translations. This would be controlled by the precurvatures already defined and thus, provides a reduced freedom to the end-effector. Moreover, path-controlling this movement would be noticeably challenging. However, monitoring all the curvatures yields more options to the manipulator to sweep 
even more space. Figure 3 a shows that changing the second tube intrinsic curvature $\kappa_{2,2}$ in the second link, provides an additional reachable zone, without even changing first and third tube translation or insertion angle. The workspace was generated with a translation sampling of $3 \mathrm{~mm}$, and a second tube curvature change sampling of $20 \mathrm{~m}^{-1}$. The workspace previously reachable by the existing active cannula type robot is hold, and an additional set of movements is achieved each time one of the curvatures is changed. It is proven by both workspaces superimposition shown in Figure $3 b$.

a)

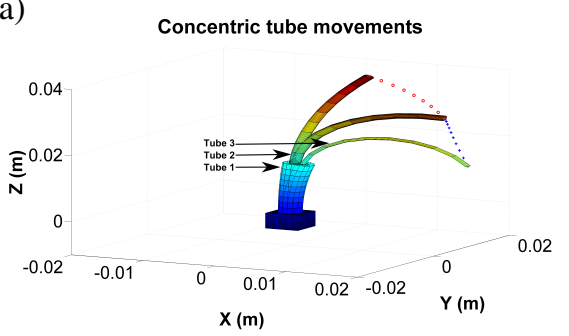

b)

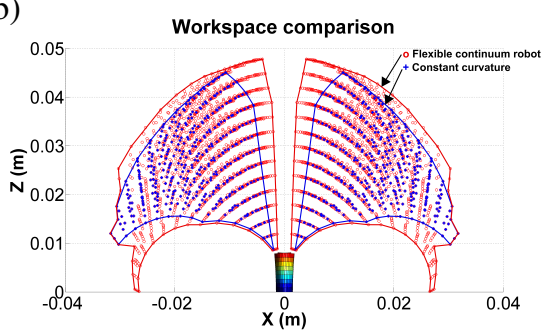

Fig. 3: End-effector workspace is marked by blue ' + ' for constant curvature active cannulas and by red 'o' for flexible continuum robot. a) Schematic description of tube translation and second tube curvature $\kappa_{2, j}$ change from 50 to $10 \mathrm{~m}^{-1}$. b) Workspaces superimposition in the $x-z$ plane.

\subsection{Performance indices}

This analysis is based on the full Jacobian $J_{\text {robot }}$ singular values $\sigma_{i}$. Thus, we need a singular-value-decomposition (SVD) of the matrix. This study is based on the three most significant performance indices: manipulability, isotropy, and condition number. Mathematical exact definitions can be found in [2] and their expressions are :

$$
\text { Manip }=\sqrt{\prod_{i} \rho_{i}}, \text { Isotropy }=\frac{\prod_{i} \rho_{i}}{\sum_{i} \rho_{i}}, \text { Condition number }=\frac{\rho_{\max }}{\rho_{\min }}
$$

As shown in Figure 4, monitoring the tube pre-curvature has a significant effect. Firstly, we notice that manipulability is enhanced when the tube bends beyond $\kappa_{2, j}=50 \mathrm{~m}^{-1}$ (Figure 4a). Otherwise, it decreases as the tube is straightened to a linear configuration. Nevertheless, the manipulator is able to reach additional zones, which was impossible with constant curvature tubes. The same phenomena is observed for the isotropy index. Straightening the tube draws the manipulator closer from a singular configuration as the isotropy reaches zero. In both cases, we notice that the isotropy measure is very low. It is due to the robot architecture that undeniably does not allow velocities in all directions similarly. Observing the third curve, the singular position is confirmed when $\kappa_{2, j}$ is near zero, with a condition 
8

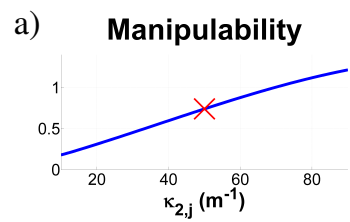

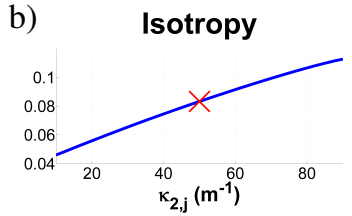

c)

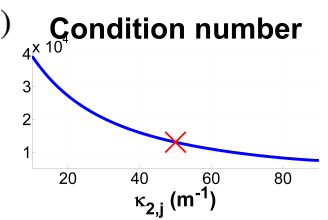

Fig. 4: Performance index variation according to second tube precurvature variation below and beyond $\kappa_{2, j}=50 \mathrm{~m}^{-1}$ fixed for the configuration in the standard approach in Section 2: a) Manipulability, b) Isotropy, and c) Condition number.

number close to infinity (Figure 4c). Beyond $\kappa_{2, j}=50 \mathrm{~m}^{-1}$, the Jacobian matrix is well-conditioned. The straight position of the manipulator is a singular configuration; thus, it is more challenging to achieve velocities. The manipulability indices are increasing with the curvatures. It is owing to the easiness to generate radial movements in a bent position.

\section{Conclusion}

In this paper, a novel EAP-based actuation technique of an active micro-endoscope was briefly described. Demonstrating the benefits of monitoring the tubes curvatures in contrast with a constant curvature existing configuration was the main contribution of this paper. On the one hand, this has been proven in terms of dependent Jacobian analysis which changes from non-holonomic to holonomic. On the other hand, performances of both manipulators have been compared through a part of the workspace as well as via the three most significant performance index evaluation. It has proven that the variable curvature improves the continuum robot performances. For the future works, additional mechanical constraints as shearing and torsion have to be included. Moreover, other tube materials that allow more flexibility need to be explored. Another challenge is to improve the actuator design: modifying the electrodes patterned along the tubes would enable more bending directions and would provide more degrees of freedom to the manipulator.

\section{Acknowledgment}

This work has been supported by the Labex ACTION project (contract ANR-11LABX-01-01) and by $\mu$ RALP, the EC FP7 ICT Collaborative Project no. 288663. 


\section{References}

1. Anderson, V.C., Horn, R.C.: Tensor arm manipulator design. ASME Transactions 1, 1-12 (1967)

2. Angeles, J., Park, F.: Performance evaluation and design criteria. In: B. Siciliano, O. Khatib (eds.) Springer Handbook of Robotics, pp. 229-244. Springer Berlin Heidelberg (2008)

3. Chirikjian, G.S., Burdick, J.W.: A modal approach to hyper-redundant manipulator kinematics. IEEE Transactions on Robotics and Automation 10(13), 343-353 (1994)

4. Gravagne, I.A., Walker, I.D.: Kinematic transformations for remotely-actuated planar continuum robots. In: IEEE International Coneference on Robotics and Automation (2000)

5. Hannan, M.W., Walker, I.D.: Kinematics and the implementation of an elephant's trunk manipulator and other continuum style robots. Journal of Robotic Systems 20(12), 45-63 (2003)

6. Murray, R.M., Li, Z., Sastry, S.S.: A Mathematical Introduction to Robotic Manipulation. CRC Press (1994). DOI 9780849379819

7. Sears, P., Dupont, P.E.: A steerable needle technology using curved concentric tubes. In: IEEE/RSJ International Conference on Intelligent Robots and Systems, pp. 2850-2856 (2006)

8. Shoa, T., Munce, N.R., Yang, V., Madden, J.D.: Conducting polymer actuator driven catheter - overview and applications. In: Proceedings of SPIE 7287, Electroactive Polymer Actuators and Devices (EAPAD), vol. 7287, pp. 1-9 (2009)

9. Webster, R.J.I., Jones, B.A.: Design and kinematic modeling of constant curvature continuum robots : A review. The International Journal of Robotics Research 29, 1661-1683 (2010)

10. Webster, R.J.I., Okamura, A.M., Cowan, N.J.: Toward active cannulas : Miniature snake-like surgical robots. In: IEEE/RSJ International Conference on Intelligent Robots and Systems, pp. $2857-2863$ (2006)

11. Webster, R.J.I., Romano, J.M., Cowan, N.J.: Mechanics of precurved-tube continuum robots. IEEE Transactions on Robotics 25(11), 67-78 (2009) 